Meta

Journal des traducteurs

Translators' Journal

\title{
Pour une combinatoire phraséologique de la publicité des droits
}

\section{Ghislaine Pesant et Estelle Thibault}

Volume 43, numéro 2, juin 1998

URI : https://id.erudit.org/iderudit/003825ar

DOI : https://doi.org/10.7202/003825ar

Aller au sommaire du numéro

Éditeur(s)

Les Presses de l'Université de Montréal

ISSN

0026-0452 (imprimé)

1492-1421 (numérique)

Découvrir la revue

Citer cet article

Pesant, G. \& Thibault, E. (1998). Pour une combinatoire phraséologique de la publicité des droits. Meta, 43(2), 328-331. https://doi.org/10.7202/003825ar

\section{Résumé de l'article}

Les auteurs démontrent la nécessité d'élargir le travail terminologique vers des vocabulaires combinatoires. Elles traitent de l'idiomaticié en langue de spécialité, de la phraséologie en langue juridique puis de leur projet de vocabulaire combinatoiredanslesous-domainedelapublicitédes droits. 


\section{BLOC-NOTES}

\section{POUR UNE COMBINATOIRE PHRASÉOLOGIQUE DE LA PUBLICITÉ DES DROITS}

\section{Résumé}

Les auteurs démontrent la nécessité d'élargir le travail terminologique vers des vocabulaires combinatoires. Elles traitent de l'idiomaticié en langue de spécialité, de la phraséologie en langue juridique puis de leur projet de vocabulaire combinatoire dans le sous-domaine de la publicité des droits.

\section{Abstract}

This article illustrates the importance of widening the scope of terminology to produce combinatory lexicons. The authors look at idiomaticity in Lsp, legal phraseology and give an overview of their combinatory lexicon project dealing with the registration of rights.

\section{[...] le langage du droit n'est pas seulement dans les termes qu'il emploie, mais aussi dans les textes qu'il produit.}

Gérard Cornu

On insiste de plus en plus, particulièrement chez les non-spécialistes d'une langue de spécialité, pour que soient élaborés des ouvrages de référence nouveaux qui soient des outils d'aide à la production (traduction, rédaction) ou à l'enrichissement des textes (révision) $)^{2}$.

Nous tenterons de voir dans cet article comment l'élargissement des travaux terminologiques peut contribuer à satisfaire cette demande, en consignant de façon systématique les unités phraséologiques propres à un discours de spécialité. Nous aborderons dans un premier point le concept d'idiomaticité en langue de spécialité, critère majeur de sélection des unités phraséologiques; un deuxième point traitera succinctement de la phraséologie juridique; dans une troisième et une quatrième partie, nous parlerons de notre projet de vocabulaire combinatoire consacré au sous-domaine de la publicité des droits en apportant quelques considérations méthodologiques et nous présenterons enfin dans une dernière partie un article en prenant pour exemple le terme inscription.

C'est en somme des premiers pas que nous accomplissons dans cette voie que nous voulons entretenir les lecteurs ici.

\section{LANGUE DE SPÉCIALITÉ ET IDIOMATICITÉ}

D'abord, qu'entendons-nous par idiomaticité en langue de spécialité ? Lorsqu'on parle d'idiomaticité on se situe d'emblée dans le système discursif de la langue, qu'il s'agisse de la langue courante ou de la langue de spécialité. En situation de production de texte au premier degré (rédaction en langue maternelle ou dans une langue autre) ou au second degré (traduction ou révision), le langagier doit viser le plus possible à produire un texte qui reflète les habitudes langagières des spécialistes du domaine qu'il traite. La situation discursive si elle déborde la terminologie ne l'exclut nullement; au contraire, le discours la met en œuvre, la met dans le texte, dans le contexte. L'idiomaticité renvoie ainsi à l'étude des critères de fonctionnement syntaxique du terme dans son réseau de cooccurrents. Pour nous, ces derniers sont des mots qui gravitent autour du terme, alors que les combinaisons plus ou moins étendues, incluant le terme, sont des phraséologismes, ceux-ci pouvant même prendre la forme d'une phrase.

Pour pouvoir parler, écrire une langue de spécialité, on ne peut donc se limiter à l'assimilation et à la maîtrise des notions et de leurs dénominations. Pour accéder au discours de spécialité, il faut d'abord en maîtriser la terminologie, mais surtout connaître certaines particularités de son fonctionnement syntaxique.

Ainsi, un texte juridique est idiomatique lorsque les juristes y reconnaissent les particularités expressives propres à leur langue; lorsqu'il reproduit exactement la terminologie du domaine mais surtout les caractéristiques qui font la spécificité de son expression linguistique. Nous éviterons de parler ici de style car, en droit, le style juridique n'est pas unique. Pensons à la langue des lois et règlements, à celle de la procédure, à celle des contrats, etc. Aussi importe-t-il de considérer le domaine d'application en cause. Le style législatif par exemple va jusqu'à comprendre la présentation. Les guides de rédaction législative ${ }^{3}$ sont notamment à cet égard très utiles.

\section{PHRASÉOLOGIE JURIDIQUE}

Mais ce qui nous intéresse plus particulièrement c'est la phraséologie, c'est-à-dire l'étude qui s'attache aux conditions d'emploi des termes dans leur contexte immédiat, aux rapports des termes avec les éléments appartenant à diverses catégories grammaticales, verbes, prépositions, adjectifs, adverbes, et non plus seule-

Meta, XLIII, 2, 1998 
ment à la catégorie nominale comme c'est le cas en terminologie classique. L'intérêt se déporte ainsi des combinaisons figées de la terminologie vers les combinaisons semi-figées propres à une langue de spécialité, en l'occurrence la langue juridique.

L'intérêt qu'il importe d'accorder à l'étude de la phraséologie juridique en droit québécois s'explique aisément du fait que celui-ci s'inspire de deux traditions juridiques, civiliste et de common law, en plus de s'exprimer dans deux systèmes linguistiques, le français et l'anglais. Ainsi, les juristes ne se trouvent pas à l'abri des influences linguistiques réciproques. Ces croisements d'un système à l'autre, d'une langue à l'autre, ne manquent pas de causer toutes sortes d'incertitudes quant à certaines formulations. C'est pourquoi les langagiers, à l'instar des juristes soucieux d'expression juste et précise, doivent constamment avoir recours à la consultation de sources dont la qualité linguistique est sûre, faute d'avoir les outils de référence adéquats.

La vérification dans les ouvrages de terminologie, lexiques ou vocabulaires, si elle renseigne sur le sens, est peu utile quant aux formulations appropriées. En outre, notre tradition lexicographique en droit est relativement récente. Le Dictionnaire de droit privé ${ }^{4}$, d'une part, est le premier ouvrage de ce type au Canada qui réponde aux normes de la terminologie. Aussi certains articles abordent la phraséologie par le biais des exemples d'emploi et des commentaires linguistiques. Mais c'est le Juridictionnaire ${ }^{5}$, qui se présente comme un «recueil des difficultés et des ressources du français juridique», qui constitue véritablement une aide à la rédaction et à la traduction. En effet il foisonne en information phraséologique bien qu'il ne soit pas un ouvrage strictement terminologique. Malheureusement l'inconvénient est que ces deux ouvrages, étant donné l'ampleur de leurs objectifs, sont encore inachevés.

\section{PROJET DE VOCABULAIRE COMBINATOIRE DE LA PUBLICITÉ DES DROITS}

Nous avons senti la grande pénurie d'ouvrages à contenu phraséologique (vocabulaires combinatoires de sous-domaines en droit), lorsque nous avons assumé, dans des délais très courts, la révision linguistique du projet de loi 125, devenu le nouveau code civil québécois. La mission fut accomplie néanmoins, mais certainement pas sans failles. Comme nous avions accumulé au cours de notre tâche un certain bagage de données de nature et terminologique et phraséologique, l'idée d'un vocabulaire combinatoire qui consignerait ces données et les enrichirait a ainsi commencé à faire son chemin. D'autant qu'un tel ouvrage permettrait de remédier dans une certaine mesure à cette lacune et de profiter aux langagiers et aux spécialistes du domaine. Nécessité faisant loi..., nous avons donc mis en œuvre notre projet. Nous avons d'abord retenu le sousdomaine de la publicité des droits, qui fait l'objet du livre IX du Code civil du Québec, compte tenu du fait que cette institution a fortement été marquée par la réforme et la nouvelle terminologie qui en a découlé. Pour ne donner qu'un exemple, le bureau d'enregistrement a été remplacé par le bureau de la publicité des droits.

\section{QUELQUES CONSIDÉRATIONS MÉTHODOLOGIQUES}

C'est donc à partir d'un point de vue résolument pratique, celui de la révision linguistique de textes, notamment législatifs ${ }^{6}$, que nous présenterons quelques considérations méthodologiques à la base de l'élaboration du Vocabulaire combinatoire de la publicité des droits.

La voie déjà tracée par Claude Lainé dans son Vocabulaire combinatoire de la CFAO nous satisfait dans l'ensemble et nous est un guide précieux. Il nous revient cependant d'adapter les caractéristiques d'un tel ouvrage au domaine juridique, notamment au sousdomaine de la publicité des droits, telle qu'elle résulte de la réforme instituée par le nouveau code civil.

La méthodologie du travail de recherche en terminologie demeure au fondement de notre pratique quotidienne de révision de textes législatifs ou juridiques en général. Par conséquent, l'objectif du terme juste à rechercher, à consigner et à traiter, est concomitant à celui de la recherche ou de la reproduction de la combinaison juste et linguistiquement correcte, appelée diversement selon les auteurs ${ }^{7}$ : groupement usuel, collocation, unité phraséologique, phraséologisme, et dont chacun des éléments constituants est un cooccurrent.

La notion de vocabulaire combinatoire évoque l'idée d'associations, de groupements. Un terme n'existe pas seul. Le terme, élément minimal de la langue de spécialité, tout comme le mot l'est dans la langue courante, n'a d'existence effective que dans ses rapports avec d'autres termes et d'autres éléments de catégories grammaticales diverses. Dans le vocabulaire traditionnel, il figure parmi d'autres termes, en premier plan dans la nomenclature, dans les index; il s'agit là de l'axe paradigmatique. Dans un vocabulaire combinatoire, le terme existe dans ses rapports avec des unités appartenant aussi à d'autres catégories que la catégorie nominale. C'est en abordant l'axe syntagmatique que nous entrons dans la phraséologie, entendue comme l'«ensemble des collocations à commutabilité restreinte, comprenant les unités terminologiques propres à un ou à plusieurs domaines de spécialité, et reflétant les habitudes langagières de leur communauté professionnelle ${ }^{8} \gg$.

Notre objectif est de procéder à la consignation des formulations usuelles dans ce sous-domaine, de façon à en avoir une image assez représentative. Le critère de sélection des unités phraséologiques est, d'une part, son idiomaticité (vue aussi comme l'écart avec la langue courante) et, d'autre part, la difficulté d'expression qu'elle présente dans le contexte de notre système bijuridique bilingue. Par exemple, quelles sont les con- 
ditions d'emploi de telle combinaison (nominale, verbale, prépositionnelle, etc.) ? Et en ce qui a trait à la présence des cooccurrents, il n'est pas toujours aisé de s'y retrouver, et de savoir exactement quelle préposition il convient d'employer avec tel nom ou après tel verbe : les prépositions $\grave{a}$, dans et $s^{9} r^{9}$ peuvent-elles accompagner indifféremment ou non le verbe publier et le verbe inscrire? Ou encore, faut-il faire usage ou non de l'article déterminé avant tel nom ou après tel verbe? (possession vaut titre, emporter présomption, avoir le droit de/ avoir droit à, etc.).

Il importe que chaque article reflète l'usage, le bon usage il va de soi, de la langue de spécialité choisie. Pour ce, doivent être présentés les quatre aspects constitutifs de la langue de spécialité, comme ils le sont d'ailleurs de la langue générale, soit: le lexique (par l'entrée), la sémantique (par la définition), la syntaxe et le style (par la liste des cooccurrents et des unités phraséologiques usuelles). Une observation à caractère normatif pourra renfermer certaines mises en garde relatives à un usage particulier à privilégier dans certains cas. On attachera aussi de l'importance à l'indication des sources (textes législatifs ou de doctrine), car il nous apparaît que le vocabulaire, qu'il se présente sur support papier ou sur support informatique, saura mieux satisfaire les besoins des spécialistes du droit et ceux des langagiers.

De plus, comme tous les termes ne s'inscrivent pas dans un réseau de cooccurrents aussi important pour les uns que pour les autres, on peut facilement prévoir que la forme des articles (d'un tel type de vocabulaire) variera en fonction des difficultés de rédaction que les unités phraséologiques présentent. Voire, la possibilité de présentation en tableaux n'est pas totalement exclue.

\section{PRÉSENTATION DE L'ARTICLE «INSCRIPTION»}

La présentation d'un vocabulaire combinatoire s'articule autour de deux axes, l'un paradigmatique, l'autre syntagmatique. Dans l'axe paradigmatique, on trouvera les unités terminologiques du sous-domaine suivies de leurs définitions. Le terme inscription figure ainsi dans la nomenclature dans un rapport associatif avec les autres termes qui forment une liste structurée présentée par ordre alphabétique. Énumérons les quelques termes suivants, assez représentatifs : bordereau d'inscription, bureau de la publicité des droits, circonscription foncière, fiche immobilière, immatriculation, inscription, officier de la publicité des droits, publicité des droits, rang, registre des droits réels et personnels, ressort, etc.

L'axe syntagmatique permet de rendre compte des particularités du discours, de la phrase et se présente sous la forme de blocs phraséologiques qui viennent enrichir l'article du vocabulaire traditionnel. Chaque terme retenu dans la nomenclature est présenté avec son réseau de cooccurrents (noms, verbes, adjectifs, adverbes, prépositions) à la suite de la ou des définitions appropriées.
Voici donc l'article INSCRIPTION tel qu'il pourrait se présenter dans un vocabulaire combinatoire de la publicité des droits. On notera d'emblée sa répartition en deux parties, la première formant le bloc terminologique et la seconde, le bloc phraséologique.

\section{INSCRIPTION $n$.f.}

1. Action d'inscrire sur un registre (officiel) ou résultat de cette opération.

2. Formalité, procédure relative à la tenue du registre.

Unités phraséologiques nominales Inscription au registre foncier de l'hypothèque Inscription d'un droit sur un registre

Inscription d'une hypothèque sur un immeuble

\section{Unités phraséologiques verbales}

Porter une inscription sur un registre

Faire une inscription sur le registre

Faire rectifier une inscription

Faire radier une inscription

Reporter une inscription sur une fiche Requérir une inscription sur le registre Inscription contestée dans les dix ans Inscription faite sous la désignation du constituant

Inscription faite sous le numéro..

Inscription faite sur le registre

Inscription requise par la présentation d'un avis

(Droit) admis à l'inscription

(Droit) soumis à l'inscription

Unités phraséologiques prépositionnelles

À compter de l'inscription

Depuis l'inscription

\author{
Autres combinaisons usuelles \\ L'inscription est réputée exacte \\ L'inscription est sans effet \\ L'inscription s'opère \\ L'inscription conserve au créancier les intérêts \\ L'inscription d'un droit emporte présomption \\ simple
}

N.B. Dans la présentation définitive, nous comptons bien sûr faire figurer la mention des sources (lois, doctrine).

\section{CONCLUSION}

Était-il vraiment nécessaire de démontrer encore ici la nécessité et l'urgence de réaliser, dans le cadre des travaux terminologiques, des vocabulaires combinatoires ? Si le principe comme tel est accepté, il reste que la pratique terminologique en est encore à ses balbutiements en ce domaine. Les questions méthodo- 
logiques sont encore en voie d'élaboration, et même les divers concepts de la phraséologie évoluent avec un certain flottement.

Nous croyons par ailleurs que c'est dans la mesure où nous aurons de plus en plus à notre disposition des postes de travail bureautique et des moyens informatiques tels que listes KWICK, logiciels de dépouillement, concordanciers, etc. que ces outils d'aide à la rédaction, à la révision et à la traduction auront la chance de voir le jour.

GHISLAINE PESANT

Office de la langue française, Montréal, Canada ESTELlE THIBAULT

Office de la langue française, Montréal, Canada

\section{Notes}

1. Gérard Cornu, Linguistique juridique, coll. «Domat droit privé», Paris, Éd. Montchrestien, 1990, p. 21.

2. Le Séminaire international sur la phraséologie organisé par le RINT, qui s'est tenu à Hull du 24 au 26 mai 1993, avait pour but de mieux cerner la notion de phraséologie, laquelle permet d'élargir le champ d'action de la terminologie.

3. Citons ici deux guides indispensables: Guide canadien de rédaction législative française, Ministère de la Justice, Ottawa et R. Tremblay, J. Lagacé et R. Journeault-Turgeon, Guide de rédaction législative, Ministère de la Justice, Québec, Soquij, 1984.

4. Centre de recherche en droit privé et comparé du Québec. Dictionnaire de droit privé et Lexiques bilingues. $2^{\mathrm{e}}$ éd., Montréal, Centre de recherche en droit privé et comparé du Québec et Les Éditions Yvon Blais inc., 1990.

5. J. Picotte, Juridictionnaire, Moncton, Centre de traduction et de terminologie juridiques de l'Université de Moncton, 1991

6. Au sujet de notre expérience de révision linguistique du Code civil du Québec, qui est en vigueur depuis le $1^{\text {er }}$ janvier 1994, voir G. Pesant et E. Thibault, «La langue des lois», Terminogramme, ${ }^{\circ}$ 61, été 1991, p. 1-3.
7. H. Béjoint et Ph. Thoiron. «Pour un index évolutif et cumulatif de cooccurrents en langue technoscientifique sectorielle», Meta, vol. 34, no 4, 1989

8. Claude Lainé, Silvia Pavel et Monique Boileau, «La phraséologie _ Nouvelle dimension de la recherche terminologique. Travaux du module canadien du RINT», Actualité terminologique, vol. 25, $\mathrm{n}^{\mathrm{o}}$ 3, 1992, p. 5 .

9. Ce sujet a été traité dans une chronique intitulée «À, dans, sur», publiée dans Le Journal du Barreau, $1^{\mathrm{er}}$ déc. 1992 , p. 14.

\section{RÉFÉRENCES}

BÉJOINT, Henri et Philippe THOIRON (1989): «Pour un index évolutif et cumulatif de cooccurrents en langue techno-scientifique sectorielle» Meta, vol. $34, \mathrm{n}^{\circ} 4$

BOILEAU, Monique, LAINÉ, Claude et Silvia PAVEL (1992): «La phraséologie - Nouvelle dimension de la recherche terminologique. Travaux du module canadien du RINT», Actualité terminologique, vol. 25, $\mathrm{n}^{\mathrm{O}} 3$, p. 5 .

Centre de recherche en droit privé et comparé du Québec (1990): Dictionnaire de droit privé et Lexiques bilingues, $2^{\mathrm{e}}$ éd., Montréal, Centre de recherche en droit privé et comparé du Québec et Les Éditions Yvon Blais inc.

CORNU, Gérard (1990) : Linguistique juridique, coll «Domat droit privé», Paris, éd. Montchrestien.

LAINÉ, Claude (1993): Vocabulaire combinatoire de la CFAO mécanique, Ottawa, RINT et Secrétariat d'État.

PESANT, Ghislaine et Estelle THIBAULT (1991): «La langue des lois», Terminogramme, Québec, Office de la langue française, $n^{\circ}$ 61, été 1991.

PESANT, Ghislaine et Estelle THIBAULT (1992): «Jurilinguistique et terminologie en discours», Terminogramme, Québec, Office de la langue française, $n^{\circ} 65$, été 1992.

PAVEL, Silvia (1993): Bibliographie de la phraséologie (1905-1992), Montréal, RINT et Secrétariat d'État.

PICOTTE, Jacques (1991): Juridictionnaire, Moncton, Centre de traduction et de terminologie juridiques de l'Université de Moncton. 